\title{
Growth of North Korean Adolescent Defectors through Temporal Acceptance and Feeling of Security about Traumatic Experience
}

\author{
Jooyoung Lee, ${ }^{1}$ Subin Park, ${ }^{2}$ and Jin Yong Jun ${ }^{1}$ \\ ${ }^{1}$ Department of Psychiatry, National Center for Mental Health, Seoul, Korea \\ ${ }^{2}$ Department of Research Planning, Mental Health Research Institute, National Center for Mental Health, Seoul, Korea
}

\author{
탈북청소년의 외상적 경험에 대한 시간적 수용과 안전감을 통한 성장 \\ 이주영 ${ }^{1}$ - 박수빈 ${ }^{2}$ - 전진용 \\ 국립정신건강센터 정신건강의학과, ${ }^{1}$ 국립정신건강센터 정신건강연구소 연구기획과 ${ }^{2}$
}

Objectives: The purpose of this study was to understand the growth of the North Korean adolescent defectors (NKAD) through temporal acceptance and feeling of security about their complex traumatic experience. Methods: We surveyed 146 NKAD, selected by two alternative schools and an NK defector clinic, to study the quality of life and related factors of the NKAD. The survey consisted of self-report questions on the satisfactory level about their past, present, and future, which follow the survey by Gallup World Poll, as well as questions about traumatic symptoms from the Child Revised Impact of Event Scale-13. Results: Subjective well-being satisfaction is higher in the future than the present or past. Also, relative satisfaction to the present is higher in the future than in the past. According to our survey, $71.9 \%$ of the NKAD have symptoms at traumatic levels, and these symptoms in the present show significant correlation only with the subject view of the past. Another significant correlation was observed between the temporal spectrum and subjective life satisfaction between the past and present $(\mathrm{r}=0.386)$, and between the present and future $(\mathrm{r}=0.587)$, but was not observed in those with traumatic symptoms. A relative view from the present to the future or to the past shows partial correlation with each temporal spectrum. Conclusion: Applying psychodynamic analysis and mind-model visualization, we sought to understand the integration of the self of the NKAD through temporal acceptance and a feeling of security, as well as the treatment approach for secure growth.

Psychoanalysis 2019;30(4):106-113

KEY WORDS: North Korean adolescent defectors · Trauma · Temporal acceptance $\cdot$ Feeling of security $\cdot$ Growth.

Received: September 11, 2019 Revised: September 23, 2019 Accepted: September 25, 2019

Address for correspondence: Jin Yong Jun, MD

Department of Psychiatry, National Center for Mental Health, 127 Yongmasan-ro, Gwangjin-gu, Seoul 04933, Korea

Tel: +82-2-2204-0151, Fax: +82-2-2204-0381, E-mail: jjy826@naver.com

\section{서 론}

시간에 대한 감각과 자아의 성장은 긴밀히 연결되어 머무 름과 흐름 등의 방향성을 갖기도 한다. 자아의 발달은 과거 와 현재 그리고 미래의 자기를 계속 유지할 수 있는 통합된 단위를 지향하는 상태이며 성장은 적응과 창조적 충동의 실 현을 의미한다(Winnicott 1965). 또한 미래의 자각은 현실의

This is an Open Access article distributed under the terms of the Creative Commons Attribution Non-Commercial License (https://creativecommons.org/licenses/by-nc/4.0) which permits unrestricted non-commercial use, distribution, and reproduction in any medium, provided the original work is properly cited.
자기 이미지와 소망하는 자기 이미지를 구분할 수 있게 되 는 과정으로 설명할 수 있다(Jacobson 1964). 탈북청소년에 게 과거는 사회환경적 변화와 외상적 경험으로 인해 생존 자체에 몰두했던 기억이며, 이로 인해 특정 내적 시간대에 머물러 있을 가능성이 높다. 그럼에도 그들은 현재에 살아가 며, 미래를 향한다. 따라서 탈북청소년의 성장에 관하여 실 제적 시간과 내적 시간의 간극을 줄이고자 하는 자아의 능 동적 관점에 기반한 이해가 필요하다.

북한이탈주민(탈북민)은 2010년 이후 급격하게 증가하여, 2018년 말 기준 약 32,000 명으로 그중 탈북청소년은 약 $11 \%$ 정도인 3,600명 정도로 추정된다(Ministry of Unification 
2018). 탈북청소년은 제 3 국에서 출생하거나 수년 내외의 성 장 기간을 거쳐, 부모와 한국에 동반 입국하거나 또는 부모 의 선입국 후 한국으로 들어왔다. 제 3 국 체류 기간 중 교육기 관에서의 재학 경험이 현저히 적고, 한국 입국 후에는 일반 정규학교 또는 대안학교에 진학하며 가족 또는 또래 그룹과 거주한다. 탈북청소년은 한국 입국 전후 언어, 문화적 차이 에 따른 적응, 양육 및 교육 환경의 변화, 불안정한 가족 관계 에 대한 노출 등 외상적 경험과 취약성이 발생할 가능성이 높다. 그 결과 성장 과정에서의 복합적인 발달 과제들이 주 어져 왔을 가능성이 높다(Lee와 Lee 2013). 일상 스트레스와 관련해서는 향후 진로와 관련한 불안이 많고, 자신에 대해 주로 무기력, 슬픔, 우울함, 흥미 저하와 같은 부정적 감정을 표현하였다. 그럼에도 대부분 스스로를 소중하게 여기는 것 으로 보였다(Korea Hana Foundation 2019).

난민 상태에서의 주요 외상과 스트레스는 그들의 공간을 떠나오는 과정, 타국으로 이동하는 과정, 그리고 정착 과정 등의 여러 단계에서 노출될 수 있다. 난민 상태에서 외상을 경험한 청소년은 발달 과정에서의 외상적 경험과 함께 가족 또는 또래 그룹 내에서 더욱 복합적 형태로 영향을 주고받 을 수 있다(Ventriglio와 Bhugra 2015). 탈북청소년은 탈북 과정 중 자신 또는 가까운 누군가의 신체적 외상의 위험성, 길어진 제 3 국에서의 체류 기간에 따른 문화 변용, 가족 해체 와 같은 외상적 사건들에 따른 심리적 취약성을 가질 수 있 다(Baek 등 2007). 그리고 그들은 탈북 과정에서 헤어진 가 족에 대한 그리움과 죄책감, 탈북민이라는 사실이 노출되는 것에 대한 두려움 등으로 인하여 불안과 우울, 외상 후 스트 레스 장애 등과 같은 심리적 고통을 지니고 있다. 외현화된 증상이 더 많을수록 지지 체계에 도움을 구하고자 하는 정 도가 낮은 것으로 보였다(Lee 2002; Kim 2013; Kim 2016; Eun과 Cho 2015). 이처럼 탈북청소년들의 삶은 그 사회 안 에서 부딪히게 되는 여러 경계들에서 자신의 존재를 드러내 고 인정받는 과정으로 볼 수 있다.

탈북청소년이 외상적 경험을 재구성하고, 극복, 회복, 그리 고 성장하는 것에 대하여, 선행연구는 정신병리적 측면과 연 관된 위험 요인에 초점이 맞춰졌다. 반면 삶의 질, 정서적 강 점과 성장 등에 대한 연구는 적었다. 난민 아동 및 청소년이 외상 경험과 문화 변용의 어려움을 비롯한 정신적 취약성을 갖고 있는 반면, 오히려 역경에 부딪혔을 때 심리적인 탄력성 이 높을 수 있는데, 이처럼 난민 청소년의 심리적 강점에 관 한 연구가 적었다(Lustig 등 2004). 저자들은 이런 배경하에 탈북청소년의 외상적 경험에 대한 과거, 현재, 미래의 시간적 수용을 정의하고, 그들의 시간적 수용과 안전감을 통한 성장 의 의미를 찾고자 하였다.

\section{대상 및 방법}

본 연구는 2017년부터 2018년까지 탈북청소년 대안학교 재학생 및 북한이탈주민 클리닉의 12 27세 탈북민 400명을 대상으로 시행한 자가 설문 평가를 바탕으로 시행했다. 연구 대상자는 12 24세 연령 범위 내 평가 항목의 누락이 없는 146명이다.

탈북청소년의 연령 범위를 규정하는 것은 아직 체계적이 지 않으며, 탈북 과정 전후의 지지 체계, 교육 과정을 기반으 로 한 심리적 성장 및 적응 과정의 고려가 필요하다(Chae 2016). 본 연구에서는 탈북청소년의 환경적, 교육적 지지의 공백 상황들을 고려하고, 북한이탈주민의 보호 및 정착 지원 에 관한 법률 시행령 제 45 조 1 항에서 필수적인 초 - 중등 교 육 지원 대상자를 만 25 세 미만의 자로 규정한 것에 따라 분 석 대상자를 12 세에서 24세 이하로 정의하였다.

평가 항목은 갤럽 월드 폴(Gallup World Poll, GWP) 5년 전 과거, 현재 그리고 5년 후 미래의 주관적 웰빙과 관련된 삶 의 만족도 평가로, 사다리 이미지에서 최악의 삶을 0 단계, 최 상의 삶을 10 단계로 체크하는 11 점 척도의 자가설문식 평가 이다(Cantril 1965). 이는 성별, 연령, 신체적, 정신적, 경제적 인 객관적 요소와 삶의 질, 사회 참여도, 안정성 등과 관련한 주관적 요소들에 대해 자율적이고 통합적인 평가를 묻는다.

한국판 아동 사건충격척도(Child Revised Impact of Event Scale-13, CRIES-13)는 현재 관찰되는 과거 외상적 경험으 로 인한 증상을 묻는 13 개 문항의 자가설문식 평가이다. CRIES-13은 Horowitz 등(1979)이 개발한 사건충격척도(The Impact of Events Scale)가 아동 대상의 평가에 적절한 문항 들로 재구성된 도구로 8세 이상의 아동을 대상으로 실시할 수 있으며, 각 문항은 4점 척도로 총점 0 에서 65점 사이에서 17점 이 posttraumatic stress disorder(PTSD)에 대한 절단점으로 확 인된 바 있다(Yule 1992; Dyregrov 등 1996). Children and War Foundation에서 한국어 자료가 제공된다. 자료 분석은 SPSS version 21.0(IBM Corp., Armonk, NY, USA)과 MATLAB version R2017a(MathWorks, Inc., Natick, MA, USA)를 이용하여 분석 및 그래프를 도출하였다. 또한 그 결 과와 관련하여 탈북청소년의 시간적 수용과 안전감을 통한 성장에 관한 정신역동적 이해를 다루었다. 연구 대상자 및 보 호자에게는 연구의 취지를 설명한 후 동의를 받았으며 이 연 구는 국립정신건강센터 임상시험심사위원회(Institutional Review Board, IRB)의 승인을 받아 이루어졌다(IRB No.: 116271-2017-11). 


\section{결 과}

\section{인구학적 특성}

대상자 146명의 평균 연령은 18.29세(standard deviation= $\pm 2.358)$ 였고, 여아의 비율이 더 높았다( $\mathrm{n}=93,63.7 \%) .2010$ 년 이후 탈북 및 한국으로 입국한 경우는 참여자 중 $90.4 \%$ 로, 2010년 이후 탈북민 수가 급증한 것을 고려했을 때 당시 상 당수의 탈북민이 자녀를 동반하여 탈북 및 한국에 입국해 오 던 상황이었음을 알 수 있다. 참여자 출생지는 북한과 중국이 각각 반을 차지하며, 부의 국적은 북한과 중국이 각각 약 $48.6 \%$ 와, $49.3 \%$, 한국이 $1.4 \%$ 인 반면, 모의 국적은 $97.3 \%$ 로 북한 국적의 비율이 월등히 높았으며, 부모의 학력은 모가 더 높은 비율(78.1\%)로 고등학교 과정 이상의 교육을 받은 것으 로 보였다. 탈북민의 장기화된 제 3 국 체류 기간에 따른 가족 형태의 변화 및 지지 체계 특성을 확인할 수 있었다(Table 1).

\section{외상적 사건에 대한 증상과 과거, 현재, 미래의 삶에 대한 주관적 만족도 평가}

$\mathrm{PTSD}$ 에 해당되는 정도의 증상이 분석 대상자의 $71.9 \%$ 를 차지하였다. 대상자 전체의 CRIES-13 평균값도 29점으로 대 체적으로 외상적 경험에 의한 증상이 동반되고 있는 것으로 보였다(Table 2,3). 또한 그들의 5년 전 과거, 현재, 5 년 후 미래에 대한 삶의 주관적 만족도는 과거나 현재에 비해 미래 에 대한 만족도가 높았다. 현재는 과거나 미래 중 낮은 만족 도를 보이는 어느 시점에 대해서든 평균적으로 약간 높은 만 족도를 보였다. 현재 시점에서 특정 시점을 바라보는 상대적 만족도는 미래를 바라볼 때 더 큰 만족도 차이를 보이며, 높 은 만족도에 집중되었다(Table 2, Figure 1)

\section{외상적 경험에 의한 현재 증상과 과거, 현재, 미래에 대한 삶의 주관적 만족도의 상관관계}

외상적 사건에 의한 현재의 PTSD 증상과 관련해서는 과
Table 1. Characteristics

\begin{tabular}{|c|c|}
\hline Variables & $\mathrm{n}=146, \mathrm{n}(\%)$ \\
\hline \multicolumn{2}{|l|}{ Gender } \\
\hline Boys & $53(36.3)$ \\
\hline Girls & $93(63.7)$ \\
\hline \multicolumn{2}{|l|}{ Age* } \\
\hline $13-18$ & $92(76.0)$ \\
\hline $19-24$ & $54(24.0)$ \\
\hline \multicolumn{2}{|l|}{ Migration } \\
\hline $2004-2009$ & $10(6.9)$ \\
\hline $2010-2018$ & $132(90.4)$ \\
\hline Missing date & $4(2.7)$ \\
\hline \multicolumn{2}{|l|}{ Repatriation } \\
\hline Yes & $11(7.5)$ \\
\hline None & $132(90.4)$ \\
\hline Missing data & $3(2.1)$ \\
\hline \multicolumn{2}{|l|}{ Birthplace } \\
\hline North Korea & $73(50.0)$ \\
\hline China & $73(50.0)$ \\
\hline \multicolumn{2}{|l|}{ Residence } \\
\hline With family & $58(39.7)$ \\
\hline With relatives & $1(0.7)$ \\
\hline With friend(s), or alone & $81(55.5)$ \\
\hline Social facilities (group-home) & $5(3.4)$ \\
\hline Missing data & $1(0.7)$ \\
\hline \multicolumn{2}{|l|}{ Nationality_father } \\
\hline North Korea & $71(48.6)$ \\
\hline China & $72(49.3)$ \\
\hline Republic of Korea & $2(1.4)$ \\
\hline \multicolumn{2}{|l|}{ Nationality_mother } \\
\hline North Korea & $143(97.3)$ \\
\hline Republic of Korea & $3(2.1)$ \\
\hline \multicolumn{2}{|l|}{ Education_father } \\
\hline High school education or more & $80(54.8)$ \\
\hline \multicolumn{2}{|l|}{ Education_mother } \\
\hline High school education or more & $114(78.1)$ \\
\hline
\end{tabular}

Table 2. Self report questionnaires value of GWP subjective well-being satisfaction and CRIES-13

\begin{tabular}{lcccc}
\hline Questionnaires score & Minimum & Maximum & Mean \pm SD & Median \\
\hline GWP past & 0 & 10 & $4.64 \pm 2.667$ & 4 \\
GWP present & 0 & 10 & $5.11 \pm 2.028$ & 5 \\
GWP future & 1 & 10 & $7.63 \pm 2.215$ & 8 \\
Present-past* $^{*}$ & -9 & 6 & $0.47 \pm 2.655$ & 1 \\
Present-future $^{\dagger}$ & -7 & 3 & $-2.52 \pm 1.889$ & -2 \\
GWP_minimum $^{\ddagger}$ & -6 & 5 & $-0.79 \pm 2.256$ & -1 \\
CRIES-13 $^{\text {G }}$ & 0 & 59 & $27.45 \pm 15.089$ & 29
\end{tabular}

*relative view from the present to the past: GWP present minus GWP past, ${ }^{\text {trelative view from the present to the future: GWP }}$ present minus GWP future, ${ }^{\ddagger} \mathrm{GWP}$ minimum $=\mathrm{MIN}$ (past, future)-present. MIN: minimum function to choose low value between two inputs. GWP: Gallup World Poll, CRIES-13: Child Revised Impact of Event Scale-13, SD: standard deviation 


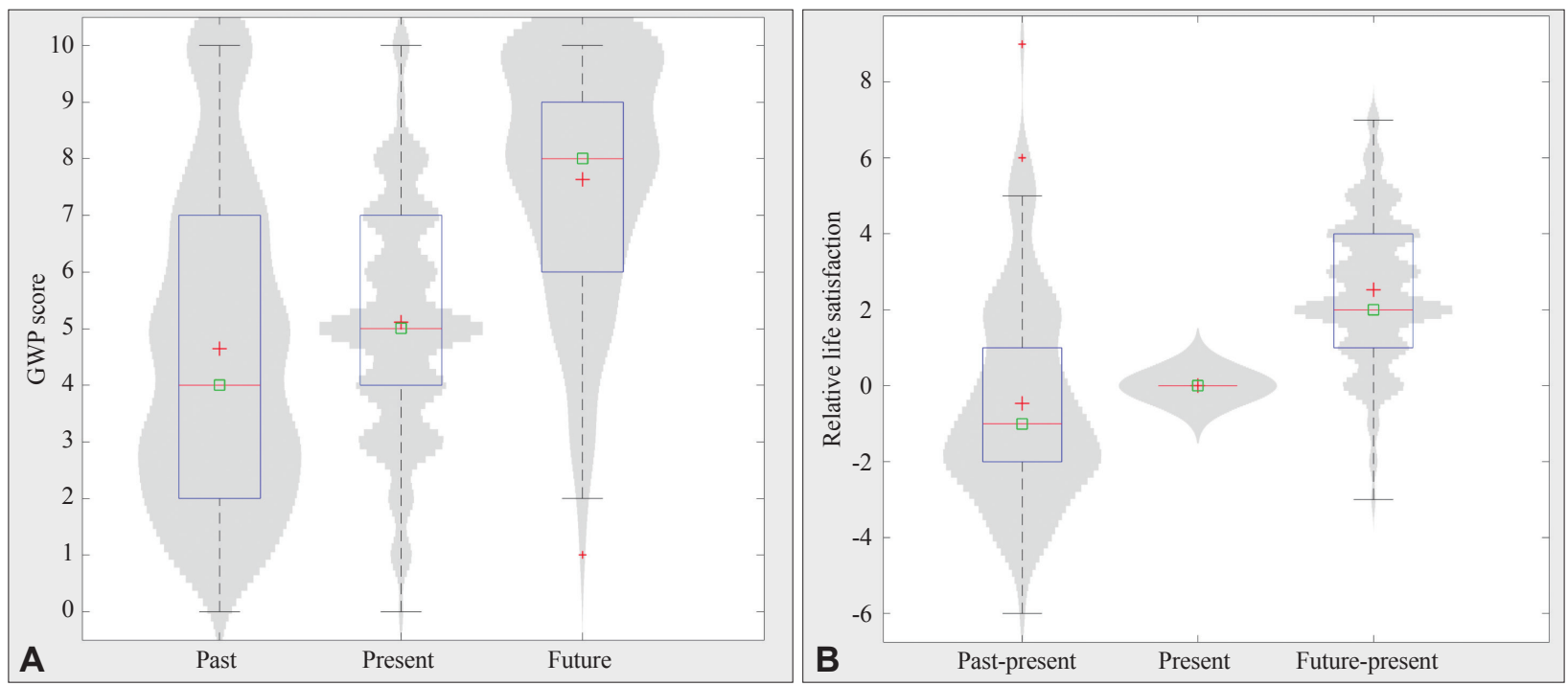

Figure 1. Plot images (box plot and violin plot) of subjective satisfaction as temporal acceptance. A: GWP subjective well-being satisfaction. B: Relative subjective satisfaction, from the view of the present. GWP: Gallup World Poll, +: mean value, $\square$ : median value.

Table 3. Relations between subjective satisfaction of life at temporal spectrum and traumatic symptoms

\begin{tabular}{lccc}
\hline $\begin{array}{c}\text { Temporal } \\
\text { spectrum }\end{array}$ & $\begin{array}{c}\text { CRIES-13 } \geq 17 \\
\mathrm{n}=105(71.9 \%)\end{array}$ & $\begin{array}{c}\text { CRIES-13<17 } \\
\mathrm{n}=41(28.1 \%)\end{array}$ & $\mathrm{p}$-value \\
\hline Past & 4.27 & 5.61 & $0.008^{*}$ \\
Present & 4.97 & 5.46 & 0.189 \\
Future & 7.63 & 7.63 & 0.998 \\
Present-past & 0.70 & -0.15 & 0.088 \\
Present-future & -2.66 & -2.17 & 0.173 \\
\hline
\end{tabular}

Values are presented as number, mean value of scale score. p-values are obtained by t-test. *statistically significant associations. p $<0.01$. CRIES-13: Child Revised Impact of Event Scale-13

거에 대한 삶의 만족도만 유의미한 차이를 보였다(Table 3). 현재 PTSD 증상의 정도와 특정 시점의 삶의 만족도 사이에 는 유의미한 상관관계가 관찰되지 않았다. 유의한 수준 내의 비교적 뚜렷한 상관관계는 현재와 과거, 현재와 미래에 대한 삶의 만족도 사이의 관계들로, 현재 시점에서 바라보는 상대 적 만족도가 기여함에 따라 상관도가 감소하였다(Table 4).

\section{고 찰}

앞선 결과에서 증상의 심각도와 관련하여 과거에 대한 삶 의 만족도만 유의미한 변수로 작용하여, 참여자의 많은 수가 현재 외상적 경험에 대한 증상과 함께 상당 부분 과거에 머 물러 있는 것으로 보였다. 미래에 대한 높은 삶의 만족도는 미래의 삶에 대해 상상하고 예상하는 탈북청소년의 자아의 확장성, 연속성 등을 의미할 수 있다. 탈북청소년이 과거와 미래를 현재와 통합하고 동시에 독립하여 바라보는 것이 그
Table 4. Correlation was noticed with temporal spectrum, subjective life satisfaction (pearson, paritial correlation analysis)

\begin{tabular}{lcc}
\hline \multicolumn{1}{c}{ Relations } & $\mathrm{r}$ & $\mathrm{p}$-value \\
\hline CRIES-13 $\leftrightarrow$ past & -0.151 & 0.068 \\
CRIES-13 $\leftrightarrow$ (present-future) $\leftrightarrow$ past & -0.145 & 0.081 \\
CRIES-13 $\leftrightarrow$ present & -0.136 & 0.101 \\
CRIES-13 $\leftrightarrow$ (present-future) $\leftrightarrow$ present & -0.130 & 0.119 \\
CRIES-13 $\leftrightarrow$ future & -0.092 & 0.271 \\
CRIES-13 $\leftrightarrow$ (past) $\leftrightarrow$ future & -0.072 & 0.387 \\
CRIES-13 $\leftrightarrow$ (present) $\leftrightarrow$ future & -0.014 & 0.863 \\
CRIES-13 $\leftrightarrow$ (present-past) $\leftrightarrow$ future & -0.112 & 0.179 \\
Past $\leftrightarrow$ present & 0.386 & $<0.01^{*}$ \\
Past $\leftrightarrow($ present-future) $\leftrightarrow$ present & 0.317 & $<0.01^{*}$ \\
Present $\leftrightarrow$ future & 0.587 & $<0.01^{*}$ \\
Present $\leftrightarrow$ (present-past) $\leftrightarrow$ future & 0.534 & $<0.01^{*}$ \\
\hline
\end{tabular}

Present-past: relative view from the present to the past. GWP present minus GWP past. Present-future: relative view from the present to the future. GWP present minus GWP future. * correlation is significant at the 0.01 level (2-tailed). GWP: Gallup World Poll, CRIES-13: Child Revised Impact of Event Scale-13

들을 충분히 현재에 머무르게 하고 또한 앞으로 나아가게 하 는 성장과 연관될 것으로 예상되었다. 한편, GWP 주관적 웰 빙 만족도 평가 척도의 특성상 사다리라는 시각적 메타포가 사용되어 평가자로 하여금 삶의 만족도에 대해 수직적 방향 성을 떠올릴 수 있다. 이는 수평적인 시각적 이미지로부터 갖게 되는 방향성과는 다르게 여겨졌을 수 있다.

\section{시간적 상실과 수용}

탈북청소년은 발달 과정에서 사회적, 그리고 대인관계 등 여러 대상적, 환경적 관계로부터 외상을 경험할 수 있다. 또 
한 탈북 전후 정착 과정 중 여러 시점에서 일시적이거나 지 속적인 정신 내외적 외상을 경험한다. 탈북청소년이 탈북 이 후 정착하게 되는 나라로 한국은 유사한 언어적 구조, 공감 대가 형성될 수 있는 역사적 배경 등의 영향으로 탈북 이후 거쳐오는 제 3 국에서의 적응 과정 및 외상적 경험 측면에서 차이가 있을 수 있다. 외상적 경험이 언어화되는 정도와 경 험하게 되는 대인관계로부터 기대되는 긴밀함 등에 있어서 도 특징적이다. 이처럼 난민 상태의 시간은 탈북청소년의 애 착관계 경험, 자기감 형성, 자아의 방어와 적응 등의 성장 과 정에 영향을 줄 수 있다. 여기서 외상 후 증상 심각도는 경험 했던 외상의 강도와 비례하지 않을 수 있다. 또한 본 연구의 결과로부터 증상의 심각도가 현재와 미래에 대한 삶의 만족 도와 독립적이라는 사실을 확인할 수 있었다. 따라서 탈북청 소년의 과거의 의미를 내적으로 대사된 외상적 경험으로서 '시간적 상실'에서 찾고자 하였다.

사람, 장소, 나라, 자유, 이상 등의 상실에 대해 Freud(1917) 는 리비도가 분리되고 다른 방향으로 향해져야 하는 상황으 로, 이때 애도 반응을 통해 상실된 대상의 내면적 연장이 이 루어진다고 하였다. Bowlby $(1961,1980)$ 는 상실한 대상과 문 화에 대한 동일시 등 다양한 반응의 가능성을 언급하여, 이를 분리 불안의 연장선으로 보기도 하였다. Ventriglio와 Bhugra (2015)는 난민의 상실과 관련하여 동시에 여러 대상들을 상 실하고 애도하는 과정으로, 외상으로 다가올 수 있고 실망 감, 지속적인 분리 불안과 슬픔, 분노 등의 표현과 함께 비상 식적인 기대감과 수동성을 보일 수 있다고 하였다. 그리고 문화적 충돌은 긴장감을 지속하게 할 수 있다고 하였다.

탈북청소년의 시간적 상실은 이중적 과정을 의미할 수 있 다. 사랑하고 가까운 이와의 시간에 대한 상실과 동시에 생 존 자체가 삶의 목적이자 소통의 수단이었던 시간으로부터 이별인 것이다. 탈북 과정에 예측할 수 없는 생존의 가능성, 삶의 안정성, 그리고 자율성 획득에 대한 기대와 함께 자아 내에서 현재와 미래에 대한 재구성이 반복되어 왔을 것이다. 탈북청소년에게 있어서 대상으로서의 과거는, 외상적 경험 으로부터 자신이 보호받지 못한 시간을 의미한다. 함입된 악 한 부모를 동일시하는 과정에서 자기 비난적인 죄의식을 가 질 수 있듯, 내재화되어 동일시된 과거는 적응의 방식에 의 해 매순간 '현재'가 되어 여러 정서를 경험하도록 하였다. 이 는 앞서 언급된 상실된 대상의 내면적 연장으로 설명할 수 있다(Freud 1917).

외상적 경험과 기억이 탈북청소년이 견뎌낼 수 있는 한계 를 넘는다면 PTSD 증상을 보일 수 있다. 탈북청소년은 대상 으로서의 과거로부터 외상적 경험을 분할하여 회피, 고립 등 의 방어기제를 보여왔으며, 외상적 기억 또한 부정, 억압과
해리 등으로 분리되어 망각, 재경험 또는 과각성되는 등 다양 한 방식으로 보일 수 있다. 이와 관련하여 Gabbard(2014)는 외상적 기억의 억압과 해리에 대해 무의식과 의식 중 어떤 의식의 수준에 머무는지, 그리고 소망이나 외상 등 무엇에 의해 동원되는지에 따른 그 차이를 언급한 바 있다.

탈북청소년은 제 3 국 또는 한국에서 문화 변용과 적응 과 정의 과제들이 주어진다. 그들에게 현재는 여전히 과거와 같 이 불안과 좌절감, 긴장을 안겨주는 대상으로서 그 연속성의 의미가 있고, 적응의 대상이 된다. 다만 그 적응의 과정이 무 엇이든, 그것은 탈북청소년이 갖는 현재와 미래에 대한 자아 의 기능이자, 그 강도를 보여주는 것이기도 하다. 저자는 탈 북청소년에게 현재는 외상 그 자체의 재경험이라기보다는, 방어기제로 인한 반복된 외상적 경험의 재현일 수 있다고 판단한다.

미래에 대한 기대와 관련해서도 방어기제의 차이를 보일 수 있다. 기대와 예상의 과정들은 탈북청소년의 자기감 형성 과 의식화 능력을 보여주는 부분이기도 하다. 하지만 일부는 통합되지 않은 과거에 대한 원시적 또는 신경증적 범주의 방어기제의 견고화 과정일 수 있다.

따라서 탈북청소년에게 있어서 시간적 수용은 대상으로서 의 시간의 의미를 의식화하고 재현하여, 미래에 대한 예상을 자발적으로 통합하는 과정을 의미한다. 이는 외상적 경험을 견딜 수 있는 좌절로 통합하고, 건강하게 도움을 구할 수 있 는 능력의 획득을 의미한다. 탈북청소년이 시간적 상실의 상 황에서 안전감을 경험한다면 시간에 대한 감각과 내적인 대 사가 이루어지고, 시간에 대한 신뢰감이 더해질 것이다.

\section{대상으로서의 시간}

탈북청소년이 특정한 관계의 사람이 아닌 시간을 대상으 로 본다는 것은, 또 그 대상으로서의 시간을 수용한다는 것 은 여러 의미를 가질 수 있다. 시간은 방향성을 갖는다. 방향 성은 시간이 대상으로서 신뢰감을 형성하게 하는 가장 일관 된 면이기도 하다. 그렇기에 시간은 통제 가능하지 않으면서 도, 그 흐름 안에서 자아가 스스로의 기능과 성장에 대해 자 발적으로 통제할 수 있기도 하다.

외상적 사건과 기억이 시간의 흐름 안에서 어떤 의미인지, 그리고 그 사건이나 기억으로 인해 어떤 자기 표상을 형성 하고 있는지 그려볼 수 있다. 결과적으로 시간을 대상으로 인식하며 외상적 기억을 좀 더 언어적으로 이해할 수 있게 된다. 언어적 기억들은 보다 제어가 가능하고, 의식적 수준 으로 받아들여진다(Gabbard 2014; Brewin 2001). 그 과정에 서 시간은 외상적 사건 속의 자신을 품어주고 안아주는 환 경(holding environment)으로 기능하며, 안전감 경험에 도 
움을 줄 수 있다. 이는 외상적 사건과 관련된 특정 대상이 함 입되어 동일시되며 자신에게 향했던 부정적 정서가 완화될 수 있음을 의미한다. 내재화된 시간은 긍정적인 대상과 부정 적 대상으로서의 통합이 이루어지며, 그 관계 안에서 자아와 대상에 대한 경험이 포괄적으로 이루어질 수 있다(Ogden 1983). 스스로 통제할 수 없었던 외상적 경험 안의 자신으로 하여금 '지금'이나 ‘내일'에 대해서는 통제할 수 있다는 감각 을 가질 수 있다. 이러한 과정은 탈북청소년이 자발적인 자 아로서 기능하고, 더 성숙한 방어기제를 보이며 자신을 이해 하는 능력을 기르는 성장을 의미한다.

\section{시간적 수용과 안전감을 통한 성장}

안전감의 경험은 Sroufe와 Waters(1977)의 '안전감의 느 낌', Ainsworth(1967)의 '안전 기지', Bowlby(1988)의 '안전 한 피난처' 개념과 같은 맥락으로 볼 수 있다.

대상으로서의 시간을 좋은 내적 관계로 경험하는 것은 시 간의 연속성을 이해하고 동시에 현재와 미래의 다양한 가능 성에 대해 신뢰감을 형성해 가는 것이라 할 수 있다. 여기서 가능성은 긍정적으로 마주할 수 있는 예상 가능성만을 의미 하는 것이 아니다. 미처 예상치 못한 정서적, 현실적 상황을 마주하더라도 이를 대상으로서의 시간 안으로 가져오는 과 정을 의미한다.

탈북청소년은 긴밀한 관계의 대상이 자주 변화될 수 있는 환경에 노출되기도 한다. 그들이 청소년기에 경험할 대상의 상실과 재애착의 과정들을 안전감으로 경험하기에 충분한 시간이 주어지지 않을 수 있다. 또한 제 3 국 출생 청소년들의 수의 증가로 외상적 경험의 경로와 강도의 변화가 동반되며 앞선 세대로부터 외상적 시간과 애착 패턴의 전이에 의한 외상적 경험의 비중이 커질 수 있다(Main 1990).

청소년은 그들의 정체성을 형성해 가는 과정 중에 있으며, 상실로부터 자신을 보호할 능력이나 누군가와 동일시할 능 력은 아직 부족할 수 있다. 하지만 본 연구의 결과로부터 탈 북청소년은 현재에 머무르고, 미래에 대한 방향성을 지니고 있음을 보였다. 미래의 자각은 대상의 다양한 측면들을 구분 하고 양가적 감정을 갖게 되고, 현실의 자기 이미지와 소망하 는 자기 이미지를 구분할 수 있게 되는 과정으로 설명할 수 있다(Jacobson 1964). Hartmann(1939)은 Freud(1911)의 현실 원리에 대해서도 기대를 포함하는 미래적 의미를 부여하며, 자아 기능으로서의 '적응'에 대해 여러 스펙트럼의 의미를 포 함하여 다루었다. 따라서 시간적 수용과 안전감을 통한 성장 은 기대, 적응, 실현의 의미들과 연결될 수 있다. 이는 자발 적으로 대상으로서의 시간에 대한 내적인 연속성을 갖는 과 정이다.
탈북청소년의 시간적 수용은 외상적 경험을 했던 과거에 서 시작된다. 그 관계 안에서 안전감을 경험한다면 대상으로 서의 시간을 충분히 감각하고 통합할 수 있는 자아를 실현 해 갈 수 있을 것이다. 실제적 시간과 내적 시간의 간극을 줄 이고자 하는 능동적 자아의 관점으로 보고자 하는 부분이기 도 하다.

\section{시간적 수용과 안전감을 통한 성장과 관련한 마음모델 제언}

외상적 사건을 통한 시간적 수용과 안전감이 자아의 성장 과 어떻게 상호작용하는지에 대한 정신역동적 이해를 마음 의 모델로 시각화하였다. 다만 여기서는 탈북청소년의 외상 적 경험의 특성을 기반으로 시간적 상실의 개념에서 출발하 였고, 보호인자나 위험인자로 언급되는 다양한 인자들과의 인과관계에 대한 적용은 제한적일 수 있다.

대상으로서의 시간은 에너지의 방향성(momentum) 개념 으로 볼 수 있다. 이때 자아가 갖는 에너지 수준의 변화는 각 각의 시점마다 그 위치를 달리할 수 있다는 점에서 '오비탈 전자구름(orbital)'(Mulliken 1932)을 메타포로 적용하여 확 률적 구름 마음모델로 표현할 수 있다. Figure 2에서 볼 수 있듯, 확률적 구름 안의 한 지점은 각 축이 의미하는 시간의 방향성 안에서 자아의 에너지 위치라 할 수 있다. 자아는 에 너지를 갖고 가속도에 의해 그 경로나 속도가 변화된 궤도 를 보일 수도 있고, 특정 시간 부근에서 정체되어 있을 수 있 다. 한 지점에 높은 확률로 머무르는 것이 결코 안정성을 의 미하지는 않는다.

Figure $2 \mathrm{~B}$ 는 자아의 에너지가 시간의 연속성과 방향성을 수용하지 못한 채 특정 과거와 연관된 영역에만 분포되어 있으며, 미래 방향으로 더 넓은 분포의 구름을 형성하지 못 한 상태를 보여준다. 그리고 원시적 또는 신경증적 방어기제 로 인해 시간을 회상하거나 재현하는 데 불안정한 경로를 보여준다. 여기서 분열, 해리 등 특정 방어기제의 공고화로 자아의 중심된 에너지 수준이 나뉘면 구름의 형태는 변화될 수도 있다. Figure $2 \mathrm{~A}$ 는 시간적 수용과 안전감을 통해 자아 의 에너지가 머무는 확률적 분포와 경로가 안정화 됨을 보여 준다. 그 범위도 시간의 방향성에 따라 자아가 예상하고 상상 가능한 수준으로 확장된다. 시각화한 확률적 구름 마음모델 에서 보여지는 바와 같이, 성장은 안전감 경험과 함께 시간 을 대상으로 수용하며, 안정화된 자아의 에너지 경로를 탄력 적으로 찾아가는 과정을 의미한다.

\section{본 연구의 한계점과 의의}

본 연구에서 이루어진 자가설문식 평가는 5년을 단위로 과 거, 현재, 미래의 삶을 평가하도록 하였다. 하지만 하루, 한 


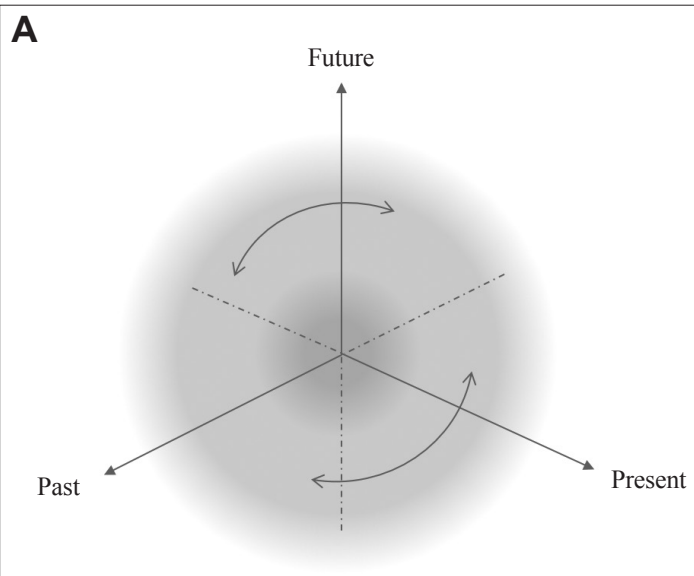

Growth through temporal acceptance and feeling of security about traumatic experience

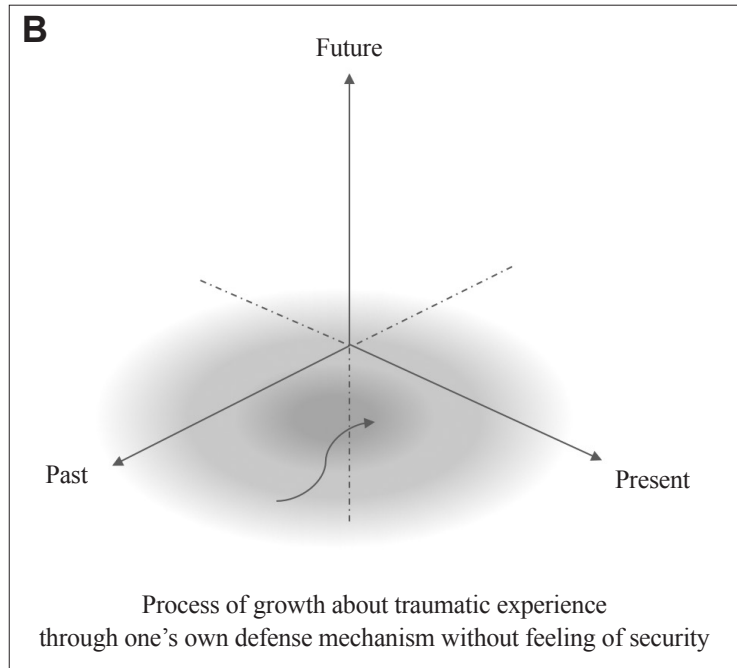

Figure 2. The probability cloud mind-model of growth through temporal acceptance and feeling of security about traumatic experience. A: Growth through temporal acceptance and feeling of security about traumatic experience. B: Process of growth about traumatic experience through one's own defense mechanism without feeling of security.

달, 10 년 등 주어진 실제적 시간에 대해서 느껴지는 주관적 거리감이 개인마다 다를 수 있을 것이다. 그리고 사회환경적 구성의 변화뿐만 아니라 SNS 등 다양한 미디어들이 탈북청 소년의 시간적 수용 및 안전감에 영향을 줄 수 있을 것으로 여겨진다. 이는 시간적, 공간적 대상관계의 변화와 함께 다 양한 정서적 변화를 동반할 수 있음을 의미한다. 앞선 제한 점이 있지만 본 연구는 탈북청소년이 충분히 현재에 머무르 고 앞으로 나아가는 성장과 관련하여 '시간적 수용'과 '안전 감'의 의미를 다루었고, 대상으로서 시간을 이해하고자 했다 는 점에서 의의가 있다.

\section{Acknowledgments}

This work was supported by a National Research Foundation of Korea (NRF) grant founded by the Korean Government (NRF-2016 R1D1A1B03931297).

\section{Conflicts of Interest}

The authors have no potential conflicts of interest to disclose.

\section{Author Contributions}

Conceptualization: Jooyoung Lee, Subin Park, Jin Yong Jun. Methodology: Jooyoung Lee, Subin Park, Jin Yong Jun. Supervision: Subin Park, Jin Yong Jun. Visualization: Jooyoung Lee. Writing — original draft: Jooyoung Lee, Subin Park, Jin Yong Jun. Writing - review \& editing: Jooyoung Lee, Subin Park, Jin Yong Jun.

\section{ORCID iDs}

Jin Yong Jun: https://orcid.org/0000-0001-7703-0505

Jooyoung Lee: https://orcid.org/0000-0002-8774-7128

\section{REFERENCES}

Ainsworth MDS. Infancy in Uganda: infant care and the growth of love. Baltimore, MD: Johns Hopkins Press;1967.
Baek HJ, Kil EB, Yoon IJ, Lee YR. A study on psychological adaptation of North Korean adolescent refugees in South Korea. Studies on Korean Youth 2007;18:183-211.

Bowlby J. Processes of mourning. Int J Psychoanal 1961;42:317-340.

Bowlby J. Attachment and loss. Vol. 3: loss, sadness and depression. New York, NY: Basic Books; 1980.

Bowlby J. A secure base: clinical applications of attachment theory. London: Routledge; 1988

Brewin CR. Memory processes in post-traumatic stress disorder. Int Rev Psychiatry 2001;13:159-163

Cantril H. The pattern of human concerns. New Brunswick, NJ: Rutgers University Press; 1965.

Chae JM. Key issues and research direction of the psychological researches for the young North Korean defectors. Korean Psychological Journal of Culture and Social Issues 2016;22:675-693.

Dyregrov A, Kuterovac G, Barath A. Factor analysis of the impact of event scale with children in war. Scand J Psychol 1996;37:339-350.

Eun J, Cho Y. A study on the perceptions of school bullying by young North Korean defectors. Studies on Korean Youth 2015;26:51-77.

Freud S. Formulations on the two principles of mental functioning. The standard edition of the complete psychological works of Sigmund Freud, Volume XII (1911-1913): the case of Schreber, papers on technique and other Works. London: Hogarth press;1911. p.213-226.

Freud S. Mourning and melancholia. The standard edition of the complete psychological works of Sigmund Freud, Volume XIV (19141916): on the history of the psycho-analytic movement, papers on metapsychology and other works. London: Horgarth press; 1917. p.237-258.

Gabbard GO. Psychodynamic psychiatry in clinical practice. Washington, DC: American Psychiatric Association Publishing;2014.

Hartmann H. Psycho-analysis and the concept of health. Int J Psa 1939; 20:308-321.

Horowitz M, Wilner N, Alvarez W. Impact of Event Scale: a measure of subjective stress. Psychosom Med 1979;41:209-218.

Jacobson E. The self and the object world. New York, NY: International Universities Press; 1964.

Kim YH. Predictors for mental health problems among young North Korean refugees in South Korea. Contemporary Society and Multiculture 2013;3:264-285

Kim YJ. Posttraumatic stress disorder as a mediator between trauma ex- 
posure and comorbid mental health conditions in North Korean refugee youth resettled in South Korea. Journal of Interpersonal Violence 2016;31:425-443.

Korea Hana Foundation [Internet]. Seoul: 2018 settlement survey of North Korean adolescent refugees in South Korea; c2019 [cited 2019 Apr 15]. Available from: https://www.koreahana.or.kr/data_room/ pro_data.jsp.

Lee KY. A qualitative study of social adaptation among North Korean refugee adolescents in South Korea. Studies on Korean Youth 2002;13: $175-223$.

Lee SH, Lee SH. Children's mental health in multicultural family and North Korean defectors in South Korea. J Korean Acad Child Adolesc Psychiatry 2013;24:124-131.

Lustig SL, Kia-Keating M, Knight WG, Geltman P, Ellis H, Kinzie JD, et al. Review of child and adolescent refugee mental health. J Am Acad Child Adolesc Psychiatry 2004;43:24-36.

Main M, Solomon J. Procedures for identifying infants as disorganized/ disoriented during the Ainsworth strange situation. In: Greenberg MT, Cicchetti D, Cummings EM. Attachment in the preschool years: theory, research, and intervention. Chicago, IL: University of Chicago Press;1990. p.121-160.

Ministry of Unification [Internet]. Seoul: Policy on North Korean Defectors; c2018 [cited 2018 May 15]. Available from: https://www.unikorea. go.kr/eng_unikorea/relations/statistics/defectors/.

Mulliken RS. Electronic structures of polyatomic molecules and valence. II. General considerations. Phys Rev 1932;41:49-71.

Ogden TH. The concept of internal object relations. Int J Psychoanal 1983;64:227-241.

Sroufe LA, Waters E. Attachment as an organizational construct. Child Development 1977;48:1184-1199.

Ventriglio A, Bhugra D. Migration, trauma and resilience. In: SchoulerOcak M. Trauma and migration cultural factors in the diagnosis and treatment of traumatised immigrants. Berlin: Springer;2015. p.69-79.

Winnicott DW. The maturational processes and the facilitating environment. London: Hogarth Press;1965.

Yule W. Post-traumatic stress disorder in child survivors of shipping disasters: the sinking of the 'Jupiter.' Psychother Psychosom 1992;57:200205. 